\title{
Internet Bandwidth Explosion: An Alternative to Submarine Cables
}

\author{
Lakshmana Kumar Yeddu \\ Freelancer \\ Airoli, Navi Mumbai, \\ Maharashtra, India
}

\author{
Shireesha Yeddu \\ Assistant Professor \\ Vivekanand Education Society's Institute of \\ Technology, Chembur, Maharashtra, India
}

\begin{abstract}
Today, Internet is known as widespread information infrastructure for its broadcasting capability, information distribution and a medium for communication between people and their computers irrespective of geographic location. From its evolution to till, Internet has transformed the dimensions of computer and communication world. An estimated one-third of the world's population is online now, a proportion that is sure to grow. More users, more devices that connect to networks and more data-heavy services to ride over the pipes are causing a "bandwidth explosion". The bandwidth demand is growing faster than the capacity to deliver it. This paper tries to address the bandwidth explosion and possible solutions.
\end{abstract}

\section{Keywords}

Bandwidth Explosion, Submarine Cables

\section{INTRODUCTION}

The Internet is a worldwide system of interconnected computer networks. The computers and computer networks exchange information using TCP/IP (Transmission Control Protocol/Internet Protocol) to communicate with each other. The computers are connected via the telecommunications networks, and the Internet can be used for e-mailing, transferring files and accessing information on the World Wide Web.

Nowadays, there are several ways that enable us to access the Internet. Technology is keep improving, method to access the Internet also increase. People can now access Internet services by using their cell phone, laptop and various gadgets. The numbers of Internet service providers are also keep increasing. For example in India, there are many Internet service providers such as BSNL, MTNL, Vodafone, Airtel, Idea etc.

Communication is becoming much easier than before due to the appearance of Internet. One of the conveniences is that messages, in the forms of email, can be sent at any corner of the world within fractions of seconds. Besides that, email also facilitated mass communication which means that one sender reaches many receivers. Some of the services made available due to Internet include video conferencing, live telecast, music, news, e-commerce, etc [1] [2].

\section{HISTORY}

In its infancy, the Internet was originally conceived by the Department of Defense as a way to protect government communications systems in the event of a military strike. The original network, dubbed ARPANet (for the Advanced Research Projects Agency that developed it) evolved into a communications channel among contractors, military personnel, and university researchers who were contributing to ARPA projects.

The network employed a set of standard protocols to create an effective way for these people to communicate and share data with each other. ARPAnet's popularity continued to spread among researchers and in the 1980 the National Science Foundation, whose NSFNet, linked several high speed computers, took charge of the what had come to be known as the Internet.

By the late 1980's, thousands of cooperating networks were participating in the Internet. In 1991, the U.S. High Performance Computing Act established the NREN (National Research \& Education Network). NREN's goal was to develop and maintain high-speed networks for research and education, and to investigate commercial uses for the Internet. The rest, as they say, is history in the making. The Internet has been improved through the developments of such services as Gopher and the World Wide Web.

Even though the Internet is predominantly thought of as a research oriented network, it continues to grow as an informational, creative, and commercial resource every day and all over the world [1] [2].

\section{INTERNET INFRASTRUCTURE}

The basic transmission infrastructure of the Internet divided into the following categories.

\subsection{Telephone / Mobile Networks}

Legacy Telephone systems are gradually being replaced by Mobile or Wireless networks for local connectivity and Optical fiber cables for physical data transmission at switching stations [3]. Though, some countries still use Dialup network connection for internet connection using local telephone wires.

With the increase in mobile phones usage, most countries turned their networks from cabling to the wireless, with the help of cell towers erected to send and receive signals especially in highways and densely populated areas. In rural areas, still underground cables or telephone cables are used to have internet usage [4].

The following Figure 1 shows the Telephone / Mobile Network Infrastructure. 


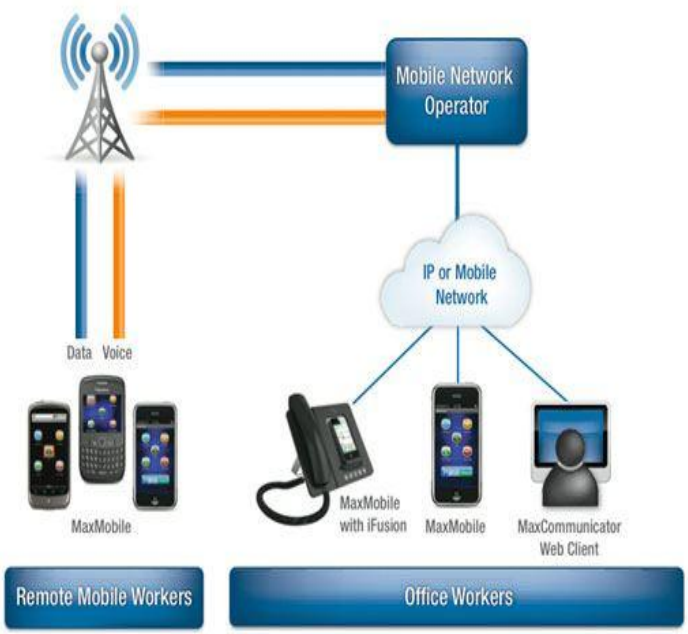

Fig 1: Telephone / Mobile Network

\subsection{Submarine Cables}

There are underground cables in the ocean, that all link together to give us a global Internet. It's really just the edges of the network where one can able to see wireless and mobile technologies."

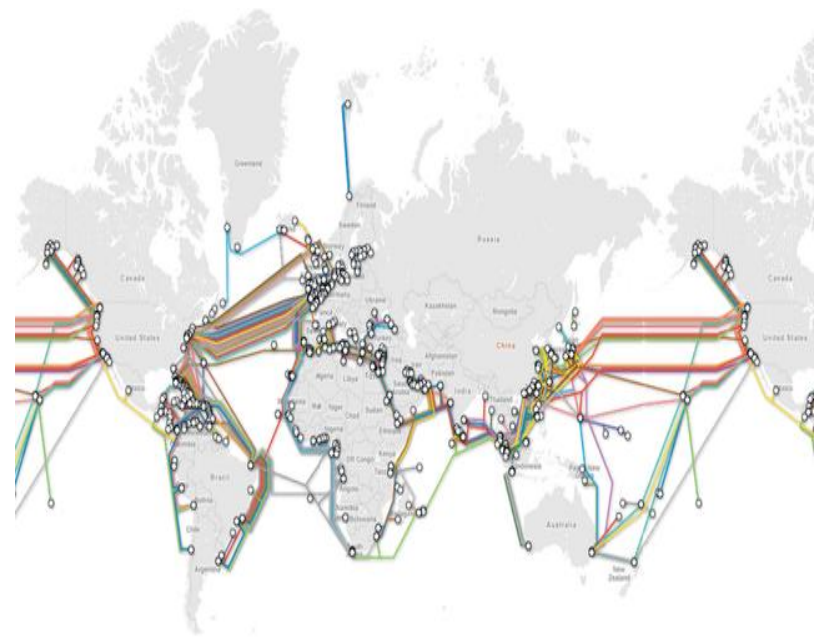

Fig 2: Submarine Cables

While the cables running under the world's oceans (see Figure 2) don't address the issue of bringing Internet capacity to farflung urban regions, they're crucial for carrying traffic between countries and continents. Underground Marine Cables are primary way that international communications happen [4].

\subsection{Satellites}

Satellites haven't been a real big part of the picture for intercontinental connectivity in quite some time. With the current usage in bandwidth via satellite is limited to just $4 \%$ which is very less and quite expensive. In-flight Wi-Fi is now accessible (see Figure 3) on around $40 \%$ of US flights and on international long haul flights via companies such as Lufthansa, Emirates and Qatar Airways. Norwegian and Turkish airlines even offer the service for free, while Scandinavian airline SAS is testing it on some of its air craft now [4].

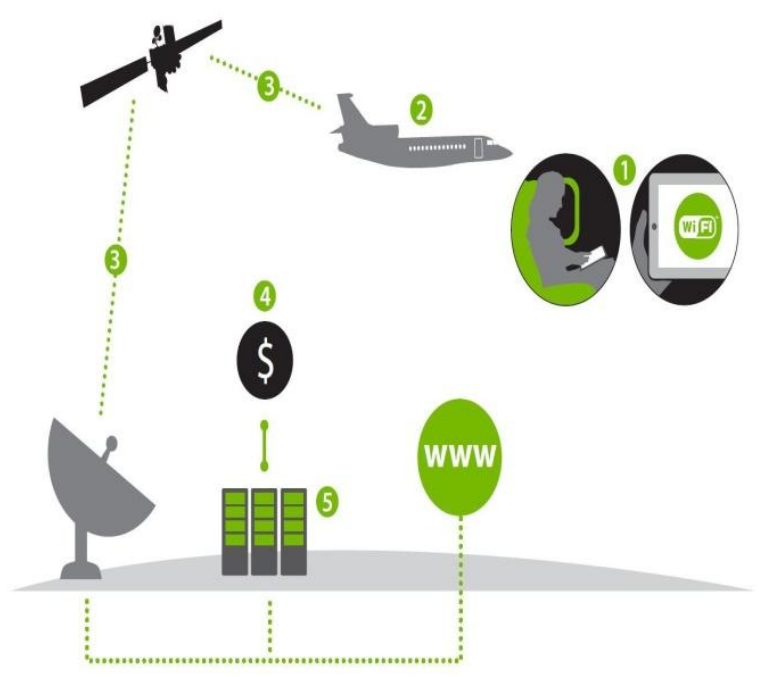

Fig 3: Satellite

\section{BANDWIDTH CAP}

Table 1 shows the top-line forecast. According to this forecast, global IP traffic in 2014 stands at 59.9 exabytes per month and will nearly triple by 2019 , to reach 168.4 exabytes per month. Consumer IP traffic will reach 138 exabytes per month and business IP traffic will surpass 29.6 exabytes per month [5].

Table 1. Global IP Traffic

\begin{tabular}{|c|c|c|c|c|c|c|c|}
\hline \multicolumn{8}{|c|}{ IPTraffic, 2014-2019 } \\
\hline & 2014 & 2015 & 2016 & 2017 & 2018 & 2019 & $\begin{array}{c}\text { CAGR } \\
2014-2019\end{array}$ \\
\hline \multicolumn{8}{|c|}{ By Type (Petabytes [PB] per Month) } \\
\hline Fixed Internet & 39,909 & 47,803 & 58,304 & 72,251 & 90,085 & 111,899 & $23 \%$ \\
\hline Managed IP & 17,424 & 20,460 & 23,371 & 26,087 & 29,274 & 31,858 & $13 \%$ \\
\hline Mobile data & 2,514 & 4,163 & 6,751 & 10,650 & 16,124 & 24,221 & $57 \%$ \\
\hline \multicolumn{8}{|c|}{ Total (PB per Month) } \\
\hline TotalIP Traffic & 59,848 & 72,426 & 88,427 & 108,988 & 135,484 & 167,978 & $23 \%$ \\
\hline
\end{tabular}

As it is seen, with the mobile users are increasing day by day, the need for speed and bandwidth is increasing exponentially.

As shown in the chart (see Figure 4) below, international bandwidth availability has soared ("used bandwidth" refers to the capacity deployed by providers, rather than bandwidth consumed by end users). From 1.4 terabits per second in 2002, it steadily climbed to 6.7 terabits in 2006 and has now reached 92.1 terabits per second. TeleGeography expects that number to hit 606.6 terabits per second in 2018 and $1,103.3$ terabits per second in 2020 [4]. 


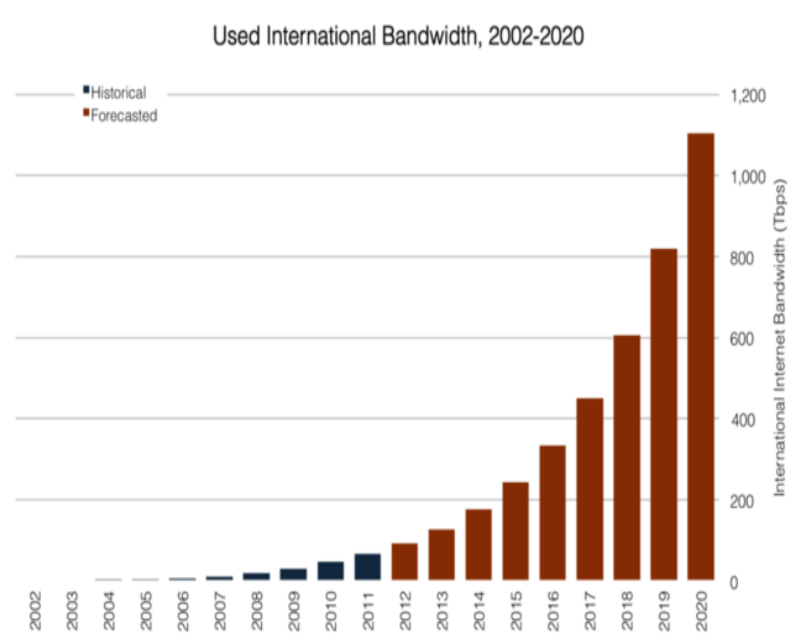

Fig 4: International Bandwidth Usage

\section{BANDWIDTH vs SPEED}

As the telecom service providers are heading to increase the speed with the increase in bandwidth by providing latest hardware to support speeds. It is evident that higher the bandwidth resulting the more speed. In some countries, the commercial speeds available are $2 \mathrm{G}, 3 \mathrm{G}, 4 \mathrm{G}$ or $5 \mathrm{G}$, at different tariff rates to limit the usage or concurrent users in support of their infrastructure.

To crub the bandwidth limitations with concurrent users reaching maximum limit, some providers are offering less low rates during the night time, to control the bandwidth usage during peak time. Online steaming is a nightmare with buffering issues, and is a concern.

As it is seen, some application are helping the users to use their services by providing Offline viewing feature to support its customers to avoid buffering issue with slow internet connection, for an example youtube offline feature.

\section{PROPOSED SOLUTIONS}

Since, the data transmission is heavily dependent on submarine cables for inter continent distribution, with the limit to the existing bandwidth of limited infrastructure, it may opt any of the following possible solutions. Appropriate cost analysis must be done before concluding the best approach.

\subsection{Airplane as Router}

Use the existing or new technology hardware or software to distribute the data transmission between continents, alternative to other networks like submarine cables or satellites. In a simple sense Airplane acts as a router or switching device (see Figure 5) or a means of hopping data(green colored path) to reach its destination.

To achieve this, it may need some international political cooperation between countries and service providers to exchange the data and may opt pay-and-use services in international flights, in the routes where there is high frequency of flights flew. It can be kept, Airplane's existing normal communication as-is without interfering proposed solution.

Maintenance of airplanes hardware is easy or can be replaced immediately, as compared to any submarine cables, due to some damage done by marine life, like Sharks or natural deterioration.

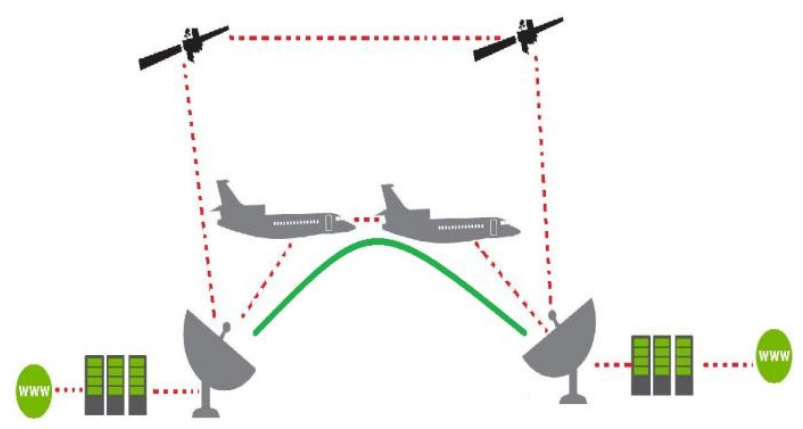

Fig 5: Airplane as Router

\subsection{Floating Towers}

Floating towers may be erected at frequent positions in seaways and the internet data may be transmitted over for inter-continental communication, shown in Figure 6, something very similar to Deep Ocean Tsunami Detection Buoys, which detect tsunami [6]. This can be a multipurpose system, like study of marine life to help fishermen, find tsunami, analysis of cyclones or even radar systems to guide seaways.

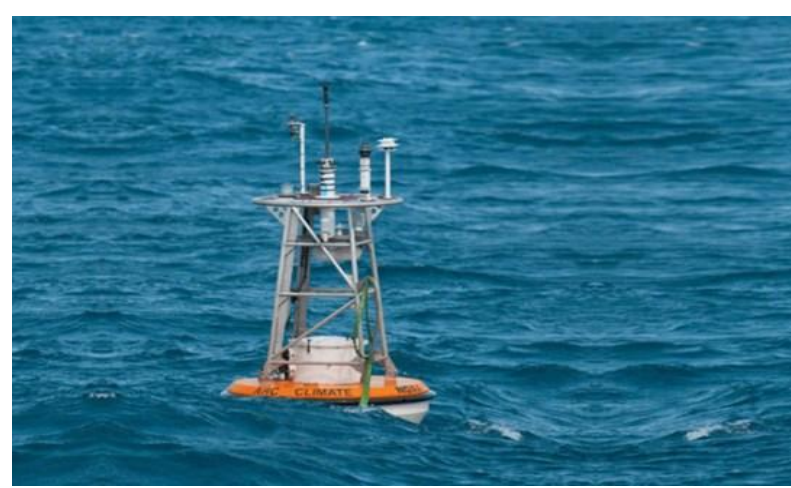

Fig 6: Floating Tower

\section{CONCLUSION}

In constant development with new cabling submarine system, or satellite communication, it is better to look for other alternative ways such as airplane as router or floating towers to support the number of concurrent users to avoid any bandwidth explosion or starvation for internet usage.

In addition, some software programs are also helping faster downloads or accessing internet. This shall add a value in effective usage of existing bandwidth. More effective programs shall be developed to support effective utilization of bandwidth of internet or routing mechanisms. Also, it requires lesser memory data formats that support Video and Audio streaming, to reduce the traffic and effective utilization of existing bandwidth, in a simple sense save some of the bandwidth for other users. Or some kind of compression techniques is needed to support the bandwidth limitation.

\section{REFERENCES}

[1] Barry M. Leiner, Vinton G. Cerf, David D. Clark, Robert E. Kahn, Leonard Kleinrock, Daniel C. Lynch, Jon Postel, Lawrence G. Roberts, Stephen S. Wolff, 2009, A Brief History of the Internet, ACM SIGCOMM Computer Communication Review, Volume 39, Number 5, October 2009 
[2] Barry M. Leiner, Vinton G. Cerf, David D. Clark, Robert E. Kahn, Leonard Kleinrock, Daniel C. Lynch, Jon Postel, Lawrence G. Roberts, Stephen S. Wolff, 1997, The Past and Future History of the INTERNET, COMMUNICATIONS OF THE ACM, February 1997/Vol. 40, No. 2.

[3] Saleh Ali K.Al-Omari, Putra Sumari, AN OVERVIEW OF MOBILE AD HOC NETWORKS FOR THE EXISTING PROTOCOLS AND APPLICATIONS, International journal on applications of graph theory in wireless ad hoc networks and sensor networks (GraphHoc), Vol.2, No.1, March 2010.

[4] Jon Brodkin, THE FUTURE OF BANDWIDTH, May 1, 2012 .

[Online]. http://arstechnica.com/business/2012/05/bandwidthexplosion-as-internet-use-soars-can-bottlenecks-beaverted/1/ [Accessed: June. 17, 2015].

[5] Cisco Visual Networking Index: Forecast and Methodology, 2014-2019 White Paper, May 27, 2015, http://www.cisco.com/c/en/us/solutions/collateral/service -provider/ip-ngn-ip-next-generation network/white_paper_c11-481360.pdf [Accessed: August. 18, 2015].

[6] Australia 2015, Bureau of Meteorology, Deep Ocean Tsunami Detection Buoys, [Online]. Available http://www.bom.gov.au/tsunami/about/detection_buoys.s html [Accessed: August. 18, 2015]. 